in many cell types. Nor is the issue of cell autonomy settled: does the expression of SPTLC1 mutations by neurons alone lead to neuropathy? The $\mathrm{C} 133 \mathrm{~W}$ transgenic mice used by Garofalo et al. (16) are not helpful in this regard, because expression of the C133W mutation is controlled by the $\beta$-actin promoter, which is ubiquitously expressed. Furthermore, even with this transgene, the amount of measured axonal loss is slight compared with the devastating loss that has been documented in affected patients (11). Let's get back to the lab.

\section{Acknowledgments}

The author's work is supported by the NIH, the National Multiple Sclerosis Society, the Muscular Dystrophy Association, and the Charcot-Marie-Tooth Association.

Address correspondence to: Steven S. Scherer, Department of Neurology, The Perelman School of Medicine at the University of Pennsylvania, Room 450 Stemmler Hall, 36th Street and Hamilton Walk, Philadelphia, Pennsylvania 19104-6077, USA.
Phone: 215.573.3198; Fax: 215.573.4454;

E-mail: sscherer@mail.med.upenn.edu.

1. Kandel ER, Schwartz JP, Jessell TM. Principles of Neural Science. New York, New York, USA: Elsevier; 2000

2. Salzer JL. Polarized domains of myelinated axons. Neuron. 2003;40(2):297-318

3. Shy ME, Lupski JR, Chance PF, Klein CJ, Dyck PJ. Hereditary motor and sensory neuropathies: an overview of clinical, genetic, electrophysiologic, and pathologic features. In: Dyck PJ, Thomas PK, eds. Peripheral Neuropathy. Philadelphia, Pennsylvania, USA: Saunders; 2005:1623-1658.

4. Timmerman V. Inherited Peripheral Neuropathies Mutation Database. Human Genome Variation Society web site. http://www.molgen.ua.ac. be/CMTMutations/. Updated February 17, 2011. Accessed September 23, 2011.

5. Latour P, et al. SIMPLE mutation analysis in dominant demyelinating Charcot-Marie-Tooth disease: three novel mutations. J Peripher Nerv Syst. 2006:11(2):148-155.

6. Saporta ASD, Sottile SL, Miller LJ, Feely SME, Siskind CE, Shy ME. Charcot-Marie-Tooth disease subtypes and genetic testing strategies. Ann Neurol. 2011;69(1):22-33

7. Scherer SS, Wrabetz L. Molecular mechanisms of inherited demyelinating neuropathies. Glia. 2008;56(14):1578-1589

8. Bejaoui K, et al. SPTLC1 is mutated in hereditary sensory neuropathy, type 1. Nat Genet. 2001; 27(3):261-262.

9. Dawkins JL, Hulme DJ, Brahmbhatt SB, Auer-
Grumbach M, Nicholson GA. Mutations in SPTLC1, encoding serine palmitoyltransferase, long chain base subunit-1, cause hereditary sensory neuropathy type I. Nat Genet. 2001;27(3):309-312.

10. Rotthier A, et al. Mutations in the SPTLC2 subunit of serine palmitoyltransferase cause hereditary sensory and autonomic neuropathy type I. Amer J Hum Genet. 2010;87(4):513-522.

11. Houlden $\mathrm{H}$, et al. Clinical, pathological and genetic characterization of hereditary sensory and autonomic neuropathy type 1 (HSAN I). Brain. 2006; 129(pt 2):411-425.

12. Nicholson GA, Dawkins JL, Blair IP, AuerGrumbach M, Brahmbhatt SB, Hulme DJ. Hereditary sensory neuropathy type I: Haplotype analysis shows founders in southern England and Europe. Amer J Hum Genet. 2001;69(3):655-659.

13. Gable K, Gupta SD, Han G, Niranjanakumari S, Harmon JM, Dunn TM. A disease-causing mutation in the active site of serine palmitoyltransferase causes catalytic promiscuity. J Biol Chem. 2010;285(30):22846-22852.

14. Penno A, et al. Hereditary sensory neuropathy type 1 is caused by the accumulation of two neurotoxic sphingolipids. J Biol Chem. 2010;285(15):11178-11187.

15. Eichler FS, et al. Overexpression of the wild-type SPT1 subunit lowers desoxysphingolipid levels and rescues the phenotype of HSAN1. J Neurosci. 2009;29(46):14646-14651

16. Garofalo K, et al. Oral L-serine supplementation reduces production of neurotoxic deoxysphingolipids in mice and humans with hereditary sensory autonomic neuropathy type 1 . J Clin Invest. 2011;121(12):4735-4745

\title{
Another VCP interactor: NF is enough
}

\author{
Conrad C. Weihl
}

Department of Neurology and Hope Center on Neurological Disorder, Washington University School of Medicine, Saint Louis, Missouri, USA.

\begin{abstract}
Inclusion body myopathy with Paget disease of the bone and frontotemporal dementia (IBMPFD) is a multisystem degenerative disorder caused by mutations in the valosin-containing protein $(V C P)$ gene. How missense mutations in this abundant, ubiquitously expressed, multifunctional protein lead to the degeneration of disparate tissues is unclear. VCP participates in diverse cellular functions by associating with an expanding collection of substrates and cofactors that dictate its functionality. In this issue of the JCI, Wang and colleagues have further expanded the VCP interactome by identifying neurofibromin-1 (NF1) as a novel VCP interactor in the CNS. IBMPFD-associated mutations disrupt binding of VCP to NF1, resulting in reduced synaptogenesis. Thus, aberrant interactions between VCP and NF1 may explain the dementia phenotype and cognitive delay observed in patients with IBMPFD and neurofibromatosis type 1.
\end{abstract}

Inclusion body myopathy with Paget disease of the bone and frontotemporal dementia (IBMPFD) is a rare, late ageonset inherited degenerative disorder that can affect the muscles, bones, and brain. It is caused by autosomal dominant muta-

Conflict of interest: The author has declared that no conflict of interest exists.

Citation for this article: J Clin Invest. 2011; 121(12):4627-4630. doi:10.1172/JCI61126. tions in the valosin-containing protein (VCP; also known as p97) gene. Although IBMPFD is a fully penetrant disorder, it has variably penetrant phenotypes (1). Approximately $50 \%$ of IBMPFD patients initially present with muscle weakness in mid-life, and more than $90 \%$ will eventually develop disabling weakness. Characteristic of the heterogeneity of this disorder, patients can initially present with a proximal or distal pattern of weakness that is often asymmet- ric. As the disease progresses, patients lose their ability to ambulate and develop respiratory failure that ultimately leads to death within 10-15 years of initial presentation. Among IBMPFD patients, 50\% develop Paget disease of the bone (PDB), manifesting as painful osteolytic lesions, and 35\% develop frontotemporal dementia (FTD), with mean onset $7-10$ years later than muscle weakness or PDB. The potential for progressing to FTD is particularly troubling to patients, who may experience changes in personality, language abilities, as well as cognitive function. The phenotypic diversity of IBMPFD is not attributable to different mutations, since two patients with the same VCP point mutation, even within the same family, can present with drastically different clinical features (e.g., dementia in one and isolated muscle weakness in another) (2). This intrafamilial variability makes the diagnosis of IBMPFD difficult. Whether there are environmental or genetic modifiers that explain this phenotypic variability is not known. 

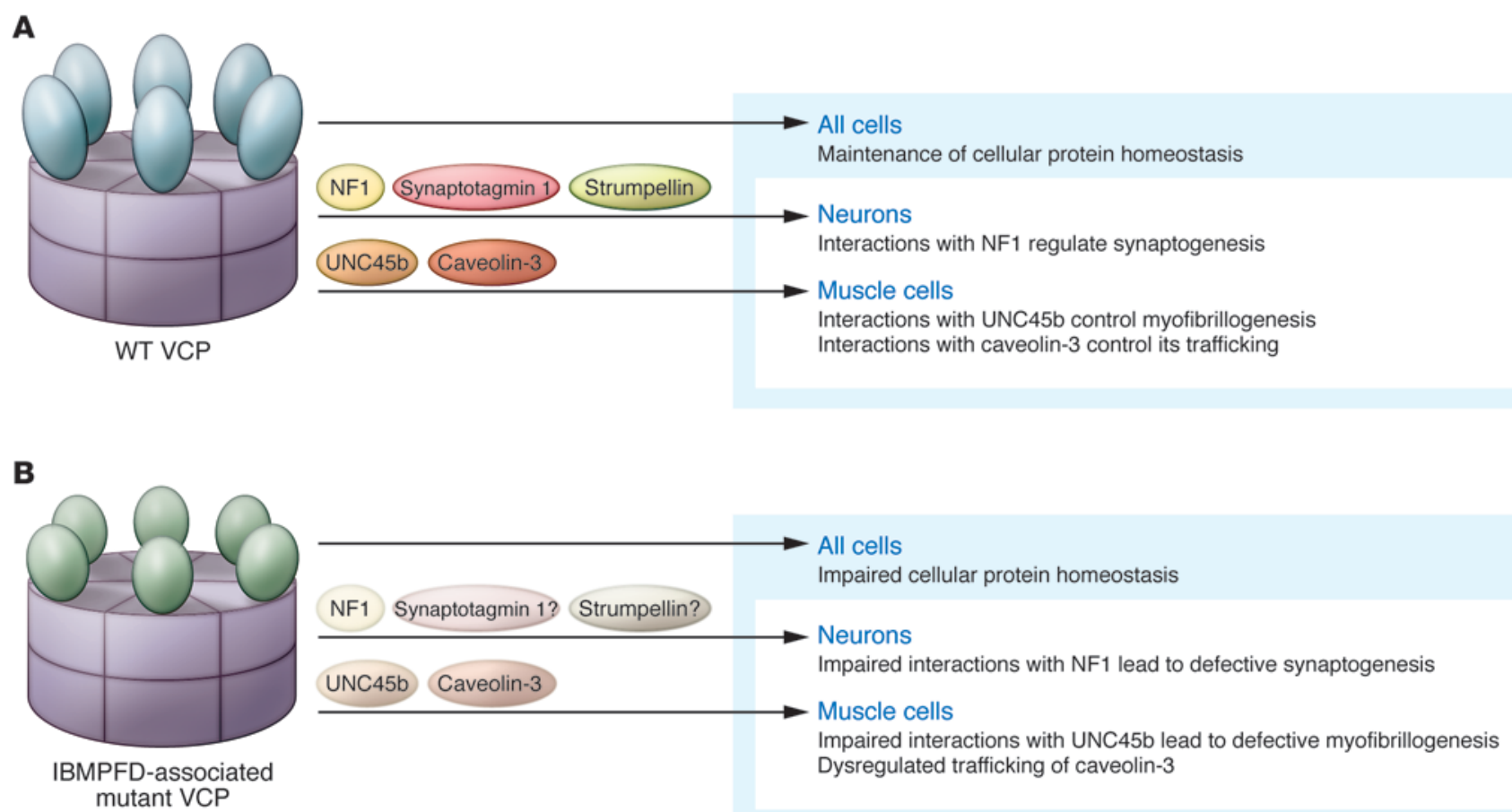

\section{Figure 1}

Function and dysfunction of VCP in health and disease. (A) VCP is necessary to maintain protein homeostasis in cells. In addition, via its association with tissue-specific substrates, VCP mediates specialized cellular processes such as synaptogenesis in neurons and myofibrillogenesis and caveolin-3 signaling in skeletal muscle. (B) IBMPFD-associated mutations in VCP disrupt protein homeostasis in VCP mutant-expressing cells, resulting in ubiquitinated inclusions, protein aggregates, and vacuolation. IBMPFD-associated VCP mutants also impair tissue-specific functions by failing to interact or regulate select substrates. This may explain the tissue-specific vulnerability in IBMPFD.

Recently, it has been established that VCP mutations are associated with neurodegenerative disorders other than IBMPFD, including amyotrophic lateral sclerosis (ALS) and Parkinson disease (PD) $(3,4)$, broadening the phenotypes caused by VCP mutations. However, even with this renewed interest in VCP and neurodegenerative disease, we do not have a clear understanding of the function of VCP in specialized cells such as neurons and how IBMPFD-associated VCP mutations alter these functions. In this issue of the JCI, Wang and colleagues now provide new insight into the function of VCP in neurons and how this is disrupted by IBMPFD-associated mutations (5). This insight could potentially explain the dementia phenotype observed in patients with IBMPFD and cognitive delay in those with neurofibromatosis type 1 .

\section{VCP, a multifunctional ATPase}

Consistent with its multiple cellular roles, VCP is an $\mathrm{AAA}^{+}$ATPase (ATPase associated with diverse cellular activities). Among the diverse cellular processes for which VCP is necessary are DNA replica- tion, mitosis, protein degradation, endocytosis, membrane fusion, and organelle biogenesis (6). The ability of VCP to participate in these functions relates to its promiscuity in substrate and cofactor binding. For example, depending upon which cofactors are available, VCP can pull aurora B kinase from chromatin to allow nuclear envelope reformation (7) or triage a misfolded protein that was extracted from the ER lumen to the proteasome for degradation (8). In order to understand the biologic processes attributed to VCP, one must therefore define its interactome. To date, more than 50 proteins have been shown to associate with VCP either directly or indirectly via ubiquitin adaptors; however, the function of many of these interactions remains unclear (9). In addition, understanding of cell type-specific interactors and cofactors that have impaired interactions with VCP mutants associated with disease is limited. Recent evidence suggests that it is alterations in cofactor and substrate association and not a global loss of VCP function that explains the pathogenesis of IBMPFD $(10,11)$.

\section{IBMPFD-associated mutations in VCP alter global protein homeostasis} In terminally differentiated cells such as the skeletal muscle cells and neurons affected in individuals with IBMPFD, VCP is important for maintaining cellular homeostasis (Figure 1A). VCP participates in the proteasomal degradation of misfolded proteins and the consolidation of protein aggregates into inclusion bodies $(8,12)$. More recently, VCP has been implicated in autophagic and lysosomal degradative pathways $(10,13)$. VCP is also necessary for the autophagic degradation of ubiquitinated proteins and mitochondria (14). In addition, VCP is required for the endolysosomal degradation of select plasma membrane proteins (10). Mutant forms of VCP associated with IBMPFD have been found to disrupt many of these homeostatic pathways (refs. 10, 12, 13, 15, and Figure 1B). IBMPFD mutations in VCP may therefore sensitize skeletal muscle and neurons to other environmental and genetic modifiers that enhance tissue-specific degeneration. Alternatively, IBMPFD-associated mutations in VCP may disrupt yet-to-be-defined tissuespecific processes accounting for the selective vulnerability of brain and skeletal muscle. 


\section{IBMPFD-associated mutations in VCP disrupt select skeletal muscle- specific processes}

In the case of myopathy, perhaps dysfunction in the skeletal muscle-specific myosin chaperone UNC45b and myosin stability $(16,17)$, as well as reduction in caveolin-3mediated signaling (10), further contribute to the muscle weakness observed in individuals with IBMPFD (Figure 1B). In skeletal muscle, IBMPFD-associated mutations in $V C P$ affect the degradation of UNC45b (16). Too much or too little UNC45b can affect the folding of myosin, altering its stability and resulting in defective myofibrillogenesis (17). VCP and its cofactor Ufd2 bind to UNC45b and facilitate its degradation via the proteasome. Loss of Ufd 2 or expression of IBMPFD-associated mutant VCP stabilizes UNC45b, subsequently altering myosin stability. The stability and function of other muscle-specific proteins may also be disrupted in the muscle of patients with IBMPFD. Using tandem affinity purification of wild-type and IBMPFD-associated mutant VCP, Ritz and colleagues identified caveolin as a novel VCP interactor that failed to associate with IBMPFD-associated mutant VCP (10). VCP forms a complex with ubiquitinated caveolin and the VCP cofactor UBXD1. Disruption of this complex by chemical inhibition of VCP, knockdown of UBXD1, or expression of IBMPFD-associated mutant VCP impairs endolysosmal trafficking of caveolin (10). In muscle from patients with IBMPFD, the muscle-specific isoform of caveolin, caveolin-3, is delocalized from the sarcolemma and accumulates within the sarcoplasm (10). Whether VCP interacts with neuronspecific substrates and if this is affected by IBMPFD-associated VCP mutations, leading to neurodegeneration, is not known.

\section{VCP interacts with NF1 in the brain and mediates synaptogenesis}

In this issue of the JCI, Wang and colleagues identify a novel VCP interactor that may help to explain the CNS phenotype in individuals with IBMPFD (Figure 1B). By immunoprecipitating neurofibromin-1 (NF1) from adult rat brain homogenates, they identified VCP, along with its cofactor p47, as highprobability interactors (5). Further analysis confirmed that VCP and NF1 interacted and mapped the region of interaction to a 400amino-acid leucine-rich domain (LRD) of NF1. In cultured rat hippocampal neurons, knockdown of VCP decreased dendritic spine density, as did NF1 depletion in mice, suggesting that they participate in the same functional pathway. Moreover, expression of the LRD region of NF1 decreased spinogenesis, presumably by interfering with the endogenous interaction between VCP and NF1, since an LRD fragment that did not associate with VCP had no effect. IBMPFDassociated mutations in VCP abrogated the interaction with NF1, and expression of IBMPFD-associated mutant VCP also diminished dendritic spine formation in cultured rat hippocampal neurons.

The mechanism by which VCP and NF1 mediate synaptogenesis is unclear. VCP associates with ubiquitinated substrates via common ubiquitin adaptor proteins that contain ubiquitin-binding domains (i.e., p47, Ufd1/Np14, UBXD1, and UBXD7) (10). Although p47 was found by Wang and colleagues to co-immunoprecipitate with VCP and NF1 in rat brain homogenates (5), it is likely not necessary for the VCP-NF1 interaction, since a truncated VCP lacking the $\mathrm{p} 47$-binding region also bound avidly to NF1. Moreover, Wang and colleagues found that neither IBMPFD-associated mutant VCP nor dominant negative VCP affected NF1 stability or ubiquitination state. This is surprising, because others have found that NF1 protein levels can be stabilized by genetic knockdown of another known VCP cofactor and NF1 interactor, UBXD8 (18). Whether UBXD8 is the missing link between VCP and NF1 in mediating synaptogenesis remains to be studied.

A role for VCP in synaptogenesis is intriguing (5). Sudhof and Sugita have previously immunoprecipitated the synaptic vesicle protein synaptotagmin 1 (Syt1) from rat brain and identified VCP as an interactor (19). VCP associates with Syt 1 in a $\mathrm{Ca}^{2+}$-dependent manner; however, unlike most specialized synaptic proteins, VCP is expressed at high levels in all tissues examined and did not increase during postnatal brain development, when synaptogenesis occurs (18). VCP has also been found to associate with another presynaptic protein, strumpellin, which is mutated in a dominant form of familial spastic paraplegia (SPG8) $(20,21)$. In Drosophila neurons, potent VCP inhibition results in cellular stress and neuronal death, while mild VCP inhibition, as might be expected with IBMPFD-associated mutant VCP expression, affects dendritic pruning, suggesting that the level of expression of VCP is important for synaptic development and maintenance (22). The study by Wang and colleagues (5) further supports the notion that VCP is necessary for synaptic development.

\section{VCP and NF1 are potential disease modifiers}

IBMPFD and neurofibromatosis type 1 are both autosomal dominant monogenic disorders that can lead to variably penetrant phenotypes in multiple organ systems. As mentioned above, patients with VCP mutations can develop pathology in muscle, brain, and bone. These pathologies vary from patient to patient, even those with the same mutation and within the same family. Similarly, patients with mutations in NF1 variably develop musculoskeletal abnormalities such as scoliosis and sphenoid wing dysplasia, along with peripheral nerve tumors such as neurofibromas and schwannomas (23). A complex constellation of cognitive deficits is also present in a vast majority of patients with neurofibromatosis type 1 (23). The genetic and/or environmental factors that modify the phenotypes of both IBMPFD and neurofibromatosis type 1 are not known. Wang and colleagues found that patients with neurofibromatosis type 1 who have mutations in the LRD region-encoding portion of the NF1 gene are more likely to have cognitive delay than those with mutations elsewhere in the NF1 gene (5). In addition, some point mutations in the LRD region-encoding portion of the NF1 gene abolished the ability of NF1 to interact with VCP, while IBMPFDassociated VCP mutants were shown to have reduced affinity for NF1. These observations place VCP and NF1 in similar pathways and suggest that alterations in their interaction may contribute to the dementia and cognitive phenotypes in IBMPFD and neurofibromatosis type 1 , respectively.

The identification of genetic modifiers for poorly penetrant monogenic disorders is critical. By manipulating a modifier of disease progression or phenotypic penetrance, one may be able to correct certain aspects of a genetic syndrome. For example, pharmacologically enhancing VCP function may be beneficial for the cognitive delay in some patients with neurofibromatosis type 1 . It is also tempting to speculate that genetic polymorphisms in the VCP or NF1 gene may influence each other's phenotype. For example, non-disease-associated polymorphisms in the LRD region of the NF1 gene may increase an IBMPFD patient's risk of developing dementia. Whether this is true remains to be determined. However, as whole-genome sequencing becomes a reality and vast amounts of genetic data are acquired from patients, it will be important to connect the genetic data with biochemical and pathway analysis. The article by Wang 
and colleagues (5) establishes a framework for how to tease out genetic and biochemical modifiers of complex phenotypes in monogenic disorders such as IBMPFD and neurofibromatosis type 1 .

\section{Acknowledgments}

Conrad C. Weihl is supported by the NIH (R01 AG031867) and the Muscular Dystrophy Association.

Address correspondence to: Conrad C. Weihl, Department of Neurology, Washington University School of Medicine, 660 S. Euclid Avenue, Saint Louis, Missouri 63110, USA. Phone: 314.362.6981; Fax: 314.362.4391; E-mail:weihlc@neuro.wustl.edu.

\footnotetext{
1. Watts GD, et al. Inclusion body myopathy associated with Paget disease of bone and frontotemporal dementia is caused by mutant valosin-containing protein. Nat Genet. 2004;36(4):377-381.

2. Weihl CC. Valosin containing protein associated fronto-temporal lobar degeneration: clinical presentation, pathologic features and pathogenesis. Curr Alzheimer Res. 2011;8(3):252-260.

3. Majounie E, et al. Mutational analysis of the VCP gene in Parkinson's disease [published online ahead of print September 13, 2011]. Neurobiol Aging. doi:10.1016/j.neurobiolaging.2011.07.011.
}

4. Johnson JO, et al. Exome sequencing reveals VCP mutations as a cause of familial ALS. Neuron. 2010;68(5):857-864.

5. Wang H-F, Shih Y-T, Chen C-Y, Chao H-W, Lee M-J, Hsueh Y-P. Valosin-containing protein and neurofibromin interact to regulate dendritic spine density. J Clin Invest. 2011;121(12):4820-4837.

6. Halawani D, Latterich M. p97: The cell's molecular purgatory? Mol Cell. 2006;22(6):713-717.

7. Ramadan K, et al. Cdc48/p97 promotes reformation of the nucleus by extracting the kinase Aurora B from chromatin. Nature. 2007;450(7173):1258-1262.

8. Rabinovich E, Kerem A, Frohlich KU, Diamant N, BarNun S. AAA-ATPase p97/Cdc48p, a cytosolic chaperone required for endoplasmic reticulum-associated protein degradation. Mol Cell Biol. 2002;22(2):626-634.

9. Dreveny I, et al. p97 and close encounters of every kind: a brief review. Biochem Soc Trans. 2004; 32(pt 5):715-720.

10. Ritz D, et al. Endolysosomal sorting of ubiquitylated caveolin-1 is regulated by VCP and UBXD1 and impaired by VCP disease mutations. Nat Cell Biol. 2011;13(9):1116-1123.

11. Fernandez-Saiz V, Buchberger A. Imbalances in $p 97$ co-factor interactions in human proteinopathy. EMBO Rep. 2010;11(6):479-485.

12. Ju JS, Miller SE, Hanson PI, Weihl CC. Impaired protein aggregate handling and clearance underlie the pathogenesis of $\mathrm{p} 97 / \mathrm{VCP}$-associated disease. J Biol Chem. 2008;283(44):30289-30299.

13. Ju JS, et al. Valosin-containing protein (VCP) is required for autophagy and is disrupted in VCP disease. J Cell Biol. 2009;187(6):875-888.

14. Tanaka A, et al. Proteasome and p97 mediate mitophagy and degradation of mitofusins induced by Parkin. J Cell Biol. 2010;191(7):1367-1380.

15. Weihl CC, Dalal S, Pestronk A, Hanson PI. Inclusion body myopathy-associated mutations in $\mathrm{p} 97 /$ VCP impair endoplasmic reticulum-associated degradation. Hum Mol Genet. 2006;15(2):189-199.

16. Janiesch PC, et al. The ubiquitin-selective chaperone CDC-48/p97 links myosin assembly to human myopathy. Nat Cell Biol. 2007;9(4):379-390.

17. Landsverk ML, et al. The UNC-45 chaperone mediates sarcomere assembly through myosin degradation in Caenorhabditis elegans. J Cell Biol. 2007;177(2):205-210.

18. Phan VT, Ding VW, Li F, Chalkley RJ, Burlingame A, McCormick F. The RasGAP proteins Ira2 and neurofibromin are negatively regulated by Gpb1 in yeast and ETEA in humans. Mol Cell Biol. 2010;30(9):2264-2279.

19. Sugita S, Sudhof TC. Specificity of Ca2+-dependent protein interactions mediated by the C2A domains of synaptotagmins. Biochemistry. 2000;39(11):2940-2949.

20. Clemen CS, et al. Strumpellin is a novel valosincontaining protein binding partner linking hereditary spastic paraplegia to protein aggregation diseases. Brain. 2010;133(10):2920-2941.

21. Valdmanis PN, et al. Mutations in the KIAA0196 gene at the SPG8 locus cause hereditary spastic paraplegia. Am J Hum Genet. 2007;80(1):152-161.

22. Rumpf S, Lee SB, Jan LY, Jan YN. Neuronal remodeling and apoptosis require VCP-dependent degradation of the apoptosis inhibitor DIAP1. Development. 2011;138(6):1153-1160.

23. Shilyansky C, Lee YS, Silva AJ. Molecular and cellular mechanisms of learning disabilities: a focus on NF1. Annu Rev Neurosci. 2010;33:221-243.

\title{
On the origin of the liver
}

\author{
Joshua R. Friedman ${ }^{1}$ and Klaus H. Kaestner ${ }^{2}$ \\ 1Department of Pediatrics, ${ }^{2}$ Department of Genetics, and Institute for Diabetes, Obesity, and Metabolism, \\ Perelman School of Medicine, University of Pennsylvania, Philadelphia, Pennsylvania, USA.
}

\begin{abstract}
While it has been well established that the fetal liver originates from foregut endoderm, the identity of the mechanisms that maintain liver mass under both basal and injury conditions remains controversial. Dramatically different models have been proposed based on the experimental design employed. In this issue of the JCI, Malato and colleagues report their elegant new model for genetic lineage tracing of mature mouse hepatocytes using an adenoassociated virus-driven Cre recombinase. They show convincingly that maintenance of liver mass during normal turnover or in response to mild injury is achieved by mature hepatocytes, rather than cholangiocytes or specialized progenitor cells, as has been suggested by others.
\end{abstract}

For parents, the question, where do babies come from? may cause dread or discomfort. The same is true for scientists who are asked, where do hepatocytes come from? The reason for this is that there is not a single answer. Hepatocytes can arise from different cell types depending on age (pre-

Conflict of interest: Klaus H. Kaestner consults for Johnson \& Johnson.

Citation for this article: J Clin Invest. 2011; 121(12):4630-4633. doi:10.1172/JCI59652. natal vs. adult), the presence or absence of hepatic injury, and even the type and degree of injury. An understanding of hepatocyte origins is a prerequisite for advancing the treatment of liver disease by promoting regeneration of endogenous hepatocytes or by deriving hepatocytes ex vivo for cellular therapy. The work of Malato and colleagues, reported in this issue of the JCI (1), adds new and important information regarding the origins of adult hepatocytes, although controversy still remains.

\section{Fetal hepatocytes are derived from hepatoblasts}

One area of clarity regarding hepatocyte origin is that embryonic and fetal hepatocytes arise from hepatoblasts, endoderm-derived cells with the potential to differentiate into either hepatocytes, which populate the bulk of the liver parenchyma, or cholangiocytes, which line the intrahepatic bile ducts. Cholangiocytes differentiate from hepatoblasts near a transient fetal structure called the ductal plate, while the remaining hepatoblasts differentiate into hepatocytes (2). Hepatoblasts are characterized by coexpression of biliary markers (e.g., cytokeratin 19) and hepatocyte markers (e.g., albumin) as well as hepatoblastspecific genes (e.g., $\alpha$-fetoprotein). Shortly after birth, this cell type is virtually undetectable in situ. This implies that the supply of hepatocytes necessary for the rapid hepatic growth that occurs in juveniles must arise from another cell type. 